\title{
La enseñanza de la cortesía en ELE: actos de habla y tendencias en investigación
}

\author{
LUCAS BRODERSEN \\ Universidad Nacional del Sur (UNS) \\ lucas.brodersen@uns.edu.ar
}

\section{Resumen}

Los aprendientes de una lengua extranjera han de desarrollar competencias que excedan los aspectos estrictamente formales para poder comunicarse de forma exitosa. Por ello, la competencia pragmática ha cobrado un rol esencial y, en este sentido, la inclusión de contenidos vinculados a la cortesía se ha tornado indispensable, tanto en los planes y programas de estudio como en el material didáctico. Este artículo ofrece un estado de la cuestión en cuanto a la producción de las numerosas investigaciones que se han llevado a cabo en los últimos años sobre el abordaje de la cortesía, más precisamente, los actos de habla, en los manuales de Español como Lengua Extranjera (ELE). Asimismo, se aborda el cuantioso número de secuencias didácticas especialmente confeccionadas para practicar los actos en contextos específicos. Consideramos que este panorama realiza considerables aportes al campo de enseñanza y aprendizaje de ELE y sirve como referencia para los estudiosos de la didáctica de la cortesía. Asimismo, se procura que sirva de incentivo para generar propuestas y definir líneas metodológicas que promuevan avances en el diseño de material.

\section{Palabras clave}

Cortesía; actos de habla; manuales; secuencias didácticas; ELE. 


\title{
The teaching of politeness in Spanish as a Foreign Language: Speech acts and tendencies in research
}

\begin{abstract}
Learners of a foreign language need to develop competences that exceed the formal aspects of a language in order to communicate in a successful way. Due to this, pragmatic competence plays an essential role and, in this sense, the inclusion of contents related to politeness has become indispensable in course programs, syllabi and teaching materials. This article offers the current status of research on the production of many research papers that have addressed, in the last years, how politeness (especially speech acts) is approached in Spanish as a Foreign Language (SFL) textbooks. In addition, we study various teaching units that have been designed to practise speech acts in specific contexts. We consider that this panorama makes substantial contributions to the teaching and learning area of SFL and helps as a reference for researchers focused on politeness. Moreover, we aim to encourage proposals and define methodological lines that promote advances in material design.
\end{abstract}

\section{Keywords}

Politeness; speech acts; textbooks; teaching units; SFL.

Recibido el 11/09/2020

Aceptado el 20/11/2020 


\section{Introducción}

La pragmática ha sido señalada como la disciplina donde los aspectos lingüísticos y los culturales encuentran su punto de convergencia. Por ello, su enseñanza posibilita la introducción de elementos culturales sin relegar lo puramente lingüístico (Dervin y Liddicoat, 2013). Del mismo modo, su aprendizaje arroja, entre otros aspectos positivos, el evitar malentendidos en contextos interculturales y la creación de estereotipos erróneos. Diversas investigaciones se han enfocado en su enseñanza y aprendizaje y han destacado la importancia de incluirla en el campo de las lenguas extranjeras (Escandell Vidal, 2004; Kasper y Rose, 2002) para que los aprendientes desarrollen la competencia pragmática. De acuerdo al Marco Común Europeo de Referencia (en adelante, MCER) (Consejo de Europa, 2002: 14), esta última se relaciona «con el uso funcional de los recursos lingüísticos (producción de funciones de lengua, de actos de habla)».

La cortesía constituye uno de los fenómenos pragmáticos más estudiados en el ámbito de Español como Lengua Extranjera (en adelante, ELE) y diversos investigadores subrayan la importancia de su incorporación en los planes de estudio y en el material didáctico (Murillo Medrano, 2004; Piatti, 2003; Urbina Vargas, 2009). Tal es así que tanto el Marco Común Europeo de Referencia (en adelante, MCER) como el Plan Curricular del Instituto Cervantes (PCIC), dos de las obras de referencia en la enseñanza de ELE, la incluyen dentro de los aspectos de la competencia comunicativa que los hablantes de una lengua extranjera han de desarrollar. El primero la incorpora dentro de las competencias sociolingüísticas que aluden a los rasgos socioculturales del uso de la lengua; el segundo la aborda en la sección Tácticas y estrategias pragmáticas, dedicada a los instrumentos que el aprendiente debe mejorar para interactuar de forma efectiva y adecuada de acuerdo al contexto y al interlocutor, y conforme a las categorías y la interpretación de situaciones y relaciones propias de los hablantes nativos (p. 251255).

Bajo estas premisas, hemos llevado a cabo una revisión de estudios sobre el abordaje de la cortesía en el material confeccionado para la enseñanza de ELE. En pos de relacionar la didáctica de la cortesía con su adquisición, nos enfocamos en investigaciones sobre un aspecto que requiere enseñanza explícita, ya sea por su difícil asimilación o porque su desconocimiento ha evidenciado problemas comunicativos entre estudiantes de ELE y hablantes nativos del español: los actos de habla. La literatura sobre el tema arroja una sustancial cantidad de trabajos que han expuesto un uso deficiente de los actos de habla en la producción de aprendientes de ELE y hablantes no nativos: el agradecimiento y la respuesta (Dumitrescu, 2006), el rechazo (Franco Martínez, 2014), la disculpa (Blázquez Castillo, 2015), las peticiones y la aceptación o rechazo de las invitaciones (Alonso Martín, 2017).

La importancia otorgada a la enseñanza de la cortesía se ha visto reflejada en su creciente incorporación al material didáctico $\mathrm{y}$, a su vez, en que numerosos investigadores se han ocupado de su abordaje. Ante el cuantioso número de valiosas publicaciones, principalmente en las últimas dos décadas, el objetivo de este trabajo es precisar el estado de la cuestión en lo que concierne a dicha producción. Este estudio realiza considerables aportes al campo de enseñanza y aprendizaje de ELE y sirve como 
referencia para los estudiosos de la didáctica de la cortesía. Asimismo, se procura que sirva de incentivo para generar propuestas y definir líneas metodológicas que promuevan avances en el diseño de material de ELE dentro del campo de la cortesía.

Para abordar el presente trabajo, luego de esta introducción ofrecemos una breve descripción teórica sobre los actos de habla (sección 2). Seguidamente, nos ocuparemos de categorizar y analizar las investigaciones sobre la didáctica de la cortesía en el ámbito de ELE (apartado 3). Finalmente, exponemos nuestras conclusiones junto con algunas sugerencias para mejorar la enseñanza de los actos de habla (sección 4).

\section{Los actos de habla}

La teoría de los actos de habla de Searle (1969) establece que es importante examinarlos porque hablar una lengua consiste, precisamente, en realizar actos de habla, como formular enunciados, dar órdenes y plantear preguntas, entre otros. El acto de habla, es decir, una oración emitida en las condiciones apropiadas, se convierte así en la unidad mínima de la comunicación lingüística y en el elemento central de la teoría.

Escandell Vidal (2004: 188), por su parte, señala que los actos de habla constituyen las unidades básicas de la comunicación. Los considera instrumentos esenciales de la pragmática que ordenan las intenciones y estudia los recursos lingüísticos que se accionan para poder expresarlas. Su clasificación de los actos se basa en el trabajo de Searle (1975) y distingue cinco tipos de acuerdo a las intenciones del emisor:

a) Asertivos (o representativos): reflejar el estado de cosas del mundo. Por ejemplo, afirmar, referir, explicar, sugerir, presumir, y quejarse.

b) Directivos: lograr que el destinatario realice una acción: ordenar, pedir, rogar, aconsejar y recomendar.

c) Compromisivos: manifestar el compromiso de llevar a cabo una acción. Comprenden actos como prometer, asegurar, garantizar, y ofrecer.

d) Expresivos: exponer el estado de ánimo sobre un tema: felicitar, agradecer, complacerse, perdonar e insultar.

e) Declarativos: producir cambios en el mundo a causa de una autoridad que se le ha otorgado. Son actos declarativos bautizar, casar, inaugurar, dictar sentencia y contratar.

Una de las taxonomías de actos de habla más utilizadas para el estudio de la cortesía del español es la propuesta por Haverkate (2004), quien los divide en corteses y no corteses. Los primeros incluyen actos expresivos (agradecer, hacer cumplidos y dar la bienvenida) y comisivos (invitar y prometer); los no corteses se dividen en descorteses (insultar) y no descorteses. Estos últimos comprenden los asertivos (aserción) y los exhortativos, que a su vez, distinguen los impositivos (ruego, súplica y mandato) de los no impositivos (consejo, recomendación e instrucción). 


\section{Estudios sobre los actos de habla en ELE}

Dentro de las publicaciones sobre la enseñanza y aprendizaje de los actos de habla en el ámbito de ELE, se distinguen numerosos trabajos que se ocupan del abordaje de los actos en los manuales, aunque también se observa una considerable cantidad de propuestas didácticas que apuntan al desarrollo de la competencia pragmática. Los manuales constituyen uno de los recursos didácticos más utilizados y sus contenidos generalmente sirven como elemento articulador de los cursos de lenguas extranjeras. Ahora bien, los docentes frecuentemente emplean diferentes materiales adicionales, entre los que se encuentran las secuencias didácticas. Así, Fernández López (2004: 724) realiza una distinción entre «manuales»y «materiales»: define a los primeros como «instrumentos de trabajo que se detienen en la presentación de todos los aspectos relacionados con la adquisición de una lengua: aspectos fonéticos y fonológicos, gramaticales, léxicos, las distintas situaciones comunicativas [...] y todo ello atendiendo a los diferentes niveles del proceso de aprendizaje de una lengua»; por otro lado, concibe a los materiales como «instrumentos complementarios que se elaboran con el fin de proporcionar al alumno y al profesor un mayor apoyo teórico o práctico relacionado con un aspecto puntual o específico del aprendizaje de la lengua».

De acuerdo a nuestra revisión bibliográfica, hemos clasificado los trabajos sobre la base de sus modalidades y objetivos:

- Investigaciones que analizan el tratamiento de actos de habla en manuales de ELE;

- Propuestas didácticas enfocadas en la enseñanza específica de uno o más actos de habla;

- Estudios que combinan el abordaje de los actos en los manuales con una propuesta o secuencia didáctica;

- Trabajos cuya descripción teórica sobre los actos de habla se acompaña con una serie de sugerencias para su enseñanza.

En los apartados siguientes, tomaremos como base esta clasificación para discutir las diversas investigaciones sobre la enseñanza de los actos de habla en el área de ELE.

\subsection{Estudios sobre los actos de habla en manuales de ELE}

Los trabajos sobre la incorporación de los actos de habla en los manuales de ELE han analizado, mayoritariamente, colecciones editadas en España que adoptan el método comunicativo y que abarcan diferentes niveles de referencia del MCER. A su vez, hemos estudiado investigaciones sobre libros de un solo nivel o que se concentran en un solo acto de habla. Igualmente, se observan estudios sobre un grupo de publicaciones editadas fuera del territorio español y otras confeccionadas para el aprendizaje de variedades diferentes a la peninsular.

En cuanto a las investigaciones que analizan diferentes actos en diversos manuales, creemos oportuno comenzar con una serie de trabajos previos al PCIC (2006), uno de los documentos de referencia de la enseñanza de ELE y cuyas directrices han marcado la confección del material didáctico en el área. Por ejemplo, la tesis doctoral de Miranda 
Ubilla (2000) - publicada incluso con anterioridad al MCER - se focaliza en la expresión de la cortesía verbal en cuatro series de manuales de español (ocho manuales en total) e inglés como lenguas extranjeras correspondientes a los niveles elemental, intermedio y avanzado ${ }^{1}$. El autor realiza un análisis estadístico sobre las estrategias que expresan cortesía (por ejemplo, tiempos verbales, funciones comunicativas, estructuras lingüísticas y direccionalidad del enunciado) y contrasta la cortesía verbal entre los distintos niveles y entre ambas lenguas. Para ello, adopta un marco pragmalingüístico con aportes de Grice (1975), Leech (1983), Brown y Levinson, (1987) y Haverkate (1994). Luego de revisar alrededor de 8000 actos, señala que la cortesía es un aspecto por lo menos periférico en la totalidad de los manuales estudiados y que merece un abordaje más intensivo.

El artículo de Bani y Nevado (2004), por su parte, se enfoca principalmente en los pronombres personales sujeto tú/usted, aunque también se ocupa del modo condicional y el modo imperativo. Desde una perspectiva pragmalingüística (Haverkate, 1994), se estudian ocho manuales editados en España e Italia destinados a adolescentes y adultos, para comprobar cómo y en qué medida hacen referencia al concepto de cortesía. Se concluye que las estrategias utilizadas para presentar la cortesía verbal y su tratamiento varían de acuerdo a los autores, el nivel, el contexto de aplicación y los objetivos de cada manual. Además, si bien todos los libros abordan los elementos lingüísticos seleccionados, ninguno ofrece explicaciones detalladas. Por último, se remarca que los ejercicios se presentan generalmente descontextualizados, porque se prioriza el aspecto formal de la lengua por sobre lo comunicativo.

El último estudio previo al PCIC es la tesis de maestría de Celia Caballero Díaz (2005), titulada «Pragmática e interculturalidad en la enseñanza del español como lengua extranjera. Las estrategias de cortesía en los manuales de E/LE». La autora estudia la forma en que se explican los consejos, las invitaciones, las órdenes, los cumplidos y la expresión de acuerdo o desacuerdo en catorce manuales que cubren desde el nivel A1 hasta el B2. Se adopta un enfoque principalmente pragmalingüístico (Haverkate, 1994) - aunque con referencia a cuestiones sociopragmáticas - y se subraya la relevancia de la cortesía como aspecto articulador entre factores lingüísticos y socioculturales. Para el análisis, se considera la contextualización de las actividades, los aspectos interculturales y la información pragmática en relación con los actos de habla. Los resultados revelan que los actos no se tratan de manera uniforme y que se priorizan los aspectos pragmalingüísticos por sobre los sociopragmáticos. De todos modos, se destaca que, en su mayoría, los manuales adoptan las propuestas interculturales planteadas en el MCER.

El abordaje de los actos en diferentes manuales y niveles se ha estudiado, ante todo, posteriormente a la publicación del PCIC. Por ejemplo, Trigo Campelo (2017) se ocupa de las peticiones, los consejos y la contraargumentación en cuatro manuales de los niveles B2 y C1 publicados con diez años de diferencia. Se analiza la frecuencia para comparar la presencia de la cortesía y se clasifican las actividades de acuerdo al tipo de

${ }_{1}^{1}$ Algunos trabajos analizan manuales previos a la publicación del MCER, por lo que en esos casos no se alude a sus niveles de referencia. 
acto y al modo en que se trabajan en cada manual. También se focaliza en la evolución del tratamiento de la cortesía, contrastando la antigüedad de los manuales. La autora concluye que los dos manuales más antiguos trabajan la cortesía con mayor profundidad desde un punto de vista cuantitativo y cualitativo, ya que sus actividades se encuadran en un contexto más adecuado y permiten distinguir la entonación de los enunciados de las actividades.

Uno de los estudios más completos sobre actos de habla en manuales de ELE es la tesis doctoral de Estela Bartol Martín (2017), titulada «La enseñanza de la cortesía verbal en ELE. Análisis y actualización de contenidos». La autora se propone evaluar el estudio y la enseñanza de la cortesía verbal en el ámbito de ELE, señalar las posibles dificultades metodológicas vinculadas a su estudio y plantear mejoras en pos de maximizar su reconocimiento y aprendizaje. Se enfatiza el aspecto social de la cortesía y la relación lengua-cultura para analizar más de veinte actos de habla en más de cuarenta manuales. Para ello, se establecen como parámetros la forma de presentación de la estrategia, cómo se explica y se vincula con el resto de los contenidos, y el tipo de actividades para su comprensión y ejercitación. Los resultados revelan un balance general positivo, dado que todos los manuales consultados trabajan, de forma más o menos explícita, aspectos de cortesía. De todos modos, generalmente no se observa un abordaje equilibrado, por lo que no es factible considerar que el tratamiento de la cortesía verbal resulte completo e integral.

Como ya mencionamos, también hemos analizado un grupo de investigaciones focalizadas en manuales de un solo nivel del MCER: tres del nivel A y una del B. En el primer caso, se destaca el artículo de Rodríguez Santos (2017) sobre los actos expresar acuerdo y desacuerdo, realizar peticiones, expresar opinión, dar instrucciones y realizar invitaciones. El objetivo del trabajo es reflexionar sobre el grado de proximidad de la confección de material didáctico a un enfoque pragmático. Dentro de un marco pragmalingüístico (Escandell Vidal, 2004), se analizan tres manuales del nivel A1 considerando el contexto de aparición de los actos y las formas lingüísticas con las que se asocian. Se concluye que, en comparación con aspectos más estructurales de la lengua, los actos reciben un tratamiento escaso y superficial en las secciones comunicativas, sumado a que no se desarrollan con precisión ni exhaustividad.

Arroyo Martínez (2014), por su parte, se enfoca en las opiniones, consejos, órdenes, expresión de deseos y peticiones en los libros de la serie Erasmus Destino: Erasmus 1 y 2. La misma está dirigida a becarios en el ámbito español y corresponde a los niveles A1 y A2. La autora enmarca su investigación en los trabajos de Haverkate (1994) y Briz Gómez (2005) y replica los criterios de análisis de Caballero Díaz (2005): descripción del manual, contextualización de las actividades, aspectos interculturales e información pragmática sobre los actos seleccionados. La valoración positiva del material se sustenta en una interesante explicación gramatical y léxica que, a su vez, se complementa con una considerable cantidad de actividades de consolidación donde se desarrollan las cuatro macrohabilidades lingüísticas de forma integrada e incentivan la reflexión intercultural.

Otra propuesta interesante es la de Fernández Martín y Núñez Cortés (2017), cuyo objetivo es comprobar la frecuencia y los contextos de aparición de los actos describir 
objetos, aconsejar, expresar certeza y evidencia, felicitar y presentar. Sobre la clasificación de actos de Escandell Vidal (2004), se examinan diez manuales del nivel A (cuatro de nivel A1, tres de nivel A2 y tres que corresponden a ambos) destinados a un público adulto. A la luz de los resultados, se señala que, si bien ciertas secuencias didácticas trabajan adecuadamente algunos actos de habla, ningún libro posibilita un aprendizaje completo y exhaustivo. Por ejemplo, no resulta claro que los manuales posibiliten al alumno deducir correctamente si un determinado acto está sujeto únicamente al contexto planteado en el manual o si puede aplicarse a otras situaciones.

Finalmente, en su tesis de maestría sobre la disculpa, Valls Anguera (2012) analiza un corpus obtenido a partir de siete manuales de los niveles B1-B2 y quince películas españolas de género realista. Por un lado, el objetivo es reconocer los contextos para disculparse en el español peninsular, atendiendo a factores como la gravedad de la ofensa y el tipo de relación entre los interlocutores. Por otro, categorizar los diferentes tipos de disculpa y comparar sus contextos de aparición con las formas lingüísticas para disculparse en los manuales y las películas. Sobre la base de un marco pragmalingüístico (Brown y Levinson, 1987, Escandell Vidal, 1996 y Haverkate, 1994), se concluye que los manuales no contienen explicaciones lingüísticas y pragmáticas suficientes y que, en general, priorizan lo gramatical por sobre lo pragmático.

Si bien las investigaciones se ocupan principalmente del abordaje de diversos actos de habla, también hemos relevado dos propuestas centradas en un solo acto. En primer lugar, destacamos el análisis de la presentación del agradecimiento en De Pablos-Ortega (2011), realizado sobre 64 manuales publicados entre 1985 y 2006 y que comprenden los niveles A1-C2 (25 de los niveles A1-A2, 20 de B1-B2 y 19 de C1-C2²). En principio, se identifica la ocurrencia de las fórmulas de agradecimiento y se las clasifica de acuerdo con una taxonomía propia. Posteriormente, se las contrasta con información obtenida de hablantes nativos del español mediante un cuestionario. El autor indica que, en su mayoría, las fórmulas de agradecimiento aparecen en los textos dialógicos, frecuentes en el enfoque basado en situaciones para introducir aspectos léxicos o gramaticales. Como aspectos negativos, señala que la cantidad y variedad de fórmulas disminuye a medida que aumenta el nivel lingüístico de los manuales (probablemente, porque en los niveles iniciales contienen un mayor número de diálogos). Asimismo, tampoco incluyen información pragmalingüística sobre el agradecimiento ni reflejan fehacientemente, de acuerdo con lo revelado en el cuestionario, los aspectos lingüísticos y socioculturales de España.

Ramajo Cuesta (2013), por su parte, estudia la aparición del cumplido en libros utilizados por estudiantes universitarios en el contexto libanés. Desde un enfoque pragmalingüístico (Haverkate, 1994; Albelda Marco, 2005), aunque incorporando también cuestiones sociopragmáticas, se concibe a este acto como una de las manifestaciones más características y recurrentes de la cortesía valorizadora. Sin embargo, al comparar quince manuales de árabe dialectal para extranjeros con 24

\footnotetext{
2 El autor señala que 38 manuales fueron publicados con anterioridad al MCER y por lo tanto, sus niveles no se correspondían con los planteados en el documento. Por ello, se les asignó un nivel de acuerdo a sus objetivos: a los que apuntaban a los niveles principiantes e intermedio bajo se los incluyó en los niveles A1-A2, a los de nivel intermedio en B1-B2, y a los niveles intermedio alto y avanzado en C1-C2.
} 
manuales de ELE correspondientes a los niveles A2 - C1, se observa que el cumplido ocupa un lugar completamente periférico, ya que apenas se recogieron muestras en los textos analizados.

La totalidad de las investigaciones hasta aquí mencionadas se enfocan en el análisis de los actos de habla en manuales publicados en España y en la variedad peninsular. Sin embargo, también se observan trabajos sobre libros producidos en otros países, como Estados Unidos, Alemania, Suecia, Corea del Sur y Argentina. En primer lugar, destacamos la tesis de maestría de Romero Betancourt (2012), donde se estudian tres manuales utilizados en el primer año del nivel universitario norteamericano (dos locales y uno español). Por un lado, se compara la frecuencia de actividades y los contenidos pragmáticos con el resto de los contenidos en cada manual. Por otro, se estudia la relación entre las formas lingüísticas de los enunciados y sus características sociales y contextuales en pos de comprobar el grado de exposición de los estudiantes a usos variados de la lengua. La autora concluye que los manuales no proporcionan input suficiente para desarrollar la competencia pragmática, dado que los contenidos pragmáticos no se presentan explícitamente y se relegan frente a otros aspectos lingüísticos. En la tesis también se propone una unidad didáctica sobre las peticiones directas e indirectas para estudiantes de un nivel intermedio alto, donde se introducen rasgos generales del español peninsular y latinoamericano y se explota el cine como recurso didáctico.

Grimm (2012), por su parte, examina cuestiones de cortesía en dos manuales utilizados por aprendientes germanoparlantes, uno editado en Alemania y otro en España. Siguiendo los parámetros del PCIC, la autora señala que abordan la cortesía de acuerdo con los niveles establecidos e incluyen información explícita sobre el comportamiento social. Por otro lado, la tesis de maestría de Patterson (2015) estudia el papel de la pragmática - más específicamente, la cortesía - en la enseñanza de ELE y el rol de la didáctica en el plan educativo del primer año del bachillerato sueco. La investigación adopta un marco pragmalingüístico (Haverkate, 1994, entre otros) con aportes de la Pragmática sociocultural (Bravo y Briz, 2004) para comparar el rol de la cortesía en tres manuales producidos en el mercado sueco con uno del mercado español. Patterson sostiene que, principalmente en los manuales suecos, se antepone el desarrollo de aspectos lingüísticos - por ejemplo, los tiempos verbales - y se relega a la cortesía a una posición periférica. Del mismo modo, resalta la falta de variación, progresión y sistematización de las actividades comunicativas orientadas al desarrollo de la conciencia pragmática.

La investigación de Rodríguez Santos y Miró Martí (2018) está enfocada en las transferencias sociopragmáticas vinculadas a los sistemas de cortesía en español y coreano en un manual editado y empleado en el ámbito surcoreano. A partir de las concepciones relativistas de la cortesía que enfatizan lo social (Bravo, 2003; Bravo y Briz, 2004) y de cómo estas se ven reflejadas en el MCER, los autores sostienen que las interferencias sociopragmáticas se originan porque los manuales asiáticos se fundamentan sobre los principios sociolingüísticos de Corea del Sur y no sobre los de las comunidades hispanohablantes. Consecuentemente, se emplean actos —como las 
peticiones y las disculpas- que pueden no ser representativos o que chocan directamente con las prácticas interaccionales de las mencionadas comunidades.

Por último, el trabajo de Brodersen (2019) estudia una serie de manuales que, además de no haber sido producidos en España, trabajan una variedad diferente a la peninsular. Se analiza la inclusión del acto saludar en ocho manuales enfocados en el español de Argentina que comprenden los niveles A1-B1. La investigación adopta un enfoque pragmalingüístico (Ferrer y Sánchez Lanza, 2002; Haverkate, 1994) con aportes de la Pragmática Sociocultural (Bravo, 2003, 2009) y atiende a las características de las actividades que ejercitan los saludos. Se concluye que, a pesar de cierta carencia de información pragmática, los manuales presentan actividades contextualizadas de forma apropiada (principalmente a través de diálogos breves). Además, incluyen una importante variedad de frases y estrategias para que el aprendiente pueda interactuar de forma exitosa en diferentes contextos comunicativos reales y con personas de distintos grupos etarios y niveles socioculturales.

A partir de nuestro análisis de los estudios enfocados en la incorporación de los actos de habla en los manuales, es posible afirmar que para el estudio de la cortesía y sus fenómenos dinamizantes, los autores adoptan, mayoritariamente, un enfoque pragmalingüístico: las investigaciones de Stephen Brown y Penélope Levinson, Henk Haverkate, y Victoria Escandell Vidal aparecen, sin lugar a dudas, entre los marcos teóricos más utilizados. De todas formas, se evidencia un creciente interés por incorporar teorías más asociadas a los estudios sociopragmáticos y relativistas de la cortesía (como la Pragmática sociocultural) en los trabajos más recientes (por ejemplo, Bartol Martín (2017), Brodersen (2019), Patterson (2015) y Rodríguez Santos y Miró Martí (2018)).

Asimismo, podemos sostener que la cortesía recibe, por lo menos, un tratamiento escaso y periférico en comparación con los contenidos formales del lenguaje. Del mismo modo, no resultan numerosas las investigaciones que realizan una valoración predominantemente positiva del tratamiento de los actos de habla, a excepción de Arroyo Martínez (2014), Bartol Martín (2017), Brodersen (2019) y Grimm (2012). En general, se considera que las actividades para trabajar los actos no se encuentran contextualizadas de forma apropiada, ni tampoco incentivan la reflexión metapragmática con asiduidad. Asimismo, se destaca que los manuales no realizan un tratamiento equilibrado de los actos, ya que, frecuentemente, se ocupan más de unos que de otros. Estas carencias se observan tanto en los trabajos publicados con anterioridad al PCIC (2006) como en los posteriores. La confluencia de estos factores indica que la enseñanza de la cortesía aún necesita perfeccionarse.

\subsection{Propuestas didácticas sobre los actos de habla}

En los últimos años, se observa una línea de investigación cuyo objetivo es ofrecer secuencias de actividades o unidades didácticas para trabajar los actos de habla en el aula. Las mismas se enmarcan en el enfoque comunicativo y adoptan diversos marcos teóricos vinculados a los estudios sobre cortesía. Además, proponen actividades sobre 
actos particulares y se confeccionan para grupos de aprendientes específicos, de acuerdo a las necesidades observadas en cada contexto.

En primer lugar, sobresalen dos investigaciones sobre los actos exhortativos que apuntan a evitar errores pragmáticos con consecuencias negativas para la interacción y la imagen del aprendiente. En la sección anterior, ya habíamos hecho referencia a la propuesta para las peticiones directas e indirectas de Romero Betancourt (2012). Otro trabajo interesante es la tesis de maestría de Caldero Cornejo (2009), donde se reconoce la importancia de los actos directivos y se ofrecen actividades de lengua en uso en pos de generar espacios de práctica.

Se observan, a su vez, dos propuestas destinadas a la enseñanza de los cumplidos y los piropos que desarrollan las cuatro macrohabilidades lingüísticas (comprensión lectora y auditiva; producción escrita y oral) de forma integrada. Martín Aizpuru y Pineda Morales (2011) ofrecen cuatro secuencias que explotan diversos recursos audiovisuales para estudiantes de los niveles B1 y B2. El objetivo radica en potenciar la reflexión metapragmática y la adquisición del vocabulario. Por su parte, la tesis de maestría de López Vila (2016) se basa en el enfoque ecléctico (que reúne aportes del método comunicativo y el estructuralismo) para el diseño de actividades que apuntan a minimizar el fallo sociopragmático en aprendientes desde el nivel inicial hasta el avanzado

Seoane y Murias (2012) trabajan los aspectos lingüísticos y gestuales vinculados al acto saludar con estudiantes universitarios pertenecientes a la cultura turca de un nivel A1-A2. Para ello, se adopta un enfoque comunicativo nocio-funcional y se proponen actividades en pares y grupales que explotan diversos recursos audiovisuales. La expresión del desacuerdo se aborda en la propuesta de Paleari (2015), sobre las bases de un enfoque pragmalingüístico contrastivo-intercultural. Las actividades buscan que los aprendientes se percaten de las diferencias culturales que se encuentran implícitas en las formulaciones lingüísticas y en las dinámicas interaccionales de las comunidades italiana y española. Para ello, mediante actividades grupales con estudiantes de diferentes L1 que estudian como L2 la lengua del otro grupo, se promueve la reflexión y la discusión sobre los enunciados y las actitudes extralingüísticas consideradas (des)corteses en una situación donde las opiniones son diferentes.

Por último, destacamos las tres secuencias para trabajar la disculpa de Jiménez et al. (2016), destinadas a estudiantes de español L1, L2 y LE. Las actividades se enmarcan en la metodología denominada Flipped Learning («Clase al revés», en español) y el enfoque por tareas y están destinadas a alumnos en situación de inmersión (L2). Se apunta a reflexionar sobre las variables culturales, evitar malentendidos mediante el reconocimiento de los contextos donde los hablantes se disculpan y aprender las expresiones de disculpa en diferentes contextos en el territorio español.

Otros autores presentan propuestas centradas en la explotación de la amplia gama de posibilidades que ofrecen los medios de comunicación como recurso didáctico. Tal es el caso de Ramos González y Rico Martín (2014), quienes examinan aspectos de cortesía en cinco series de Radiotelevisión Española (RTVE) transmitidas por Internet. Las autoras realizan un análisis de carácter dual: por un lado, un estudio cualitativo que aborda la expresión de la cortesía a través de aspectos lingüísticos, paralingüísticos, 
quinésicos, proxémicos y cronémicos de los actos de habla; por otro, un análisis cuantitativo que pone de manifiesto la presencia de estos elementos en las series analizadas. La cortesía es enmarcada dentro de los contenidos socioculturales y se selecciona este tipo de series accesibles a través de Internet, ya que se considera factible trabajar con material similar proveniente de regiones hispanohablantes diferentes a la peninsular. Asimismo, los estudiantes son expuestos a interacciones reales de una variedad de registros en situaciones comunicativas que proporcionan herramientas para adecuar su idiolecto al contexto situacional.

Posteriormente, en su tesis doctoral, Ramos González (2015) ofrece otra secuencia a partir de las ya mencionadas series de RTVE. Dentro de un marco pragmalingüístico (Haverkate, 1994) y con aportes de la Pragmática sociocultural (Bravo, 2004, 2005), el objetivo central consiste en observar la actitud de los estudiantes frente a las actividades propuestas y promover la reflexión sobre si les ayudarían a evitar un fallo pragmático. Se elaboran actividades comunicativas, graduadas de acuerdo al nivel lingüístico de los estudiantes, y adecuadas al contexto universitario israelita. El análisis se complementa, a su vez, con tests de hábitos sociales, el diario de la profesora, cuestionarios de autoevaluación de los alumnos y de evaluación de la propuesta didáctica. Los resultados muestran, en primer lugar, la presencia de rasgos de cortesía en las series de televisión. Además, si bien la mayoría de los estudiantes reconoce que la propuesta didáctica les amplió el conocimiento sobre aspectos de la cortesía, la cultura propia y la española, no aseguran que podrían evitar el fallo pragmático en una situación comunicativa real.

Para finalizar, destacamos la unidad didáctica en la tesis de maestría de Martínez Guillén (2018), quien sobre las bases del enfoque por tareas, utiliza películas españolas para trabajar la expresión de opiniones.

Las propuestas didácticas que acabamos de mencionar demuestran un esfuerzo progresivo, en la última década, por mejorar la competencia pragmática mediante la adecuación de la enseñanza de los actos de habla para aprendientes de ELE de niveles y contextos específicos. En general, las secuencias están planteadas para estudiantes del ámbito universitario en diversos espacios geográficos: Turquía, Estados Unidos, Italia, Grecia y Francia.

Nuestra valoración de las unidades revisadas resulta sumamente positiva por diversos factores. En primer lugar, aspiran a desarrollar la competencia pragmática mediante la integración uniforme de las cuatro macrohabilidades lingüísticas. Además, adoptan el enfoque comunicativo y el enfoque por tareas, priorizando así la enseñanza de los actos a través de actividades de lengua en uso por sobre lo estructural. Asimismo, las propuestas cubren el espectro total de los niveles de referencia del MCER, es decir, desde los iniciales hasta los más avanzados. Del mismo modo, explotan una interesante cantidad y variedad de actividades (principalmente, los juegos de rol) y recursos didácticos (mayoritariamente, los medios de comunicación). Por último, destacamos que no solo se concentran en la enseñanza de cuestiones vinculadas a los elementos pragmalingüísticos de los actos de habla, sino que han incorporado la enseñanza de los rasgos sociopragmáticos de forma progresiva. Esto implica que ya no se priorizan 
únicamente los aspectos estructurales, sino que también se considera el contexto de emisión de los enunciados o la familiaridad y la jerarquía entre los interlocutores.

\subsection{Estudios sobre los actos de habla en manuales de habla que incluyen propuestas didácticas}

En este apartado, nos centraremos en una serie de investigaciones que, a partir de las carencias observadas en relación al abordaje de los actos de habla en manuales de ELE, proponen secuencias didácticas destinadas a mejorar la competencia pragmática. Aquí distinguimos el trabajo de Martín Aizpuru (2010), donde a partir de la escasez de fórmulas rutinarias sociales (Corpas Pastor, 1997) observada en nueve manuales entre los niveles A1-C1, se presenta una propuesta para las funciones agradecer, responder a un agradecimiento, disculparse y responder a una disculpa. Se sugiere una fase de observación, donde se familiariza al aprendiente con las funciones comunicativas, y una fase de consolidación, en la cual se ahonda en las características fraseológicas de las fórmulas empleadas.

Hemos de destacar también el trabajo de Zerva (2013), cuya visión no universalista de la cortesía reconoce que las estructuras y formas lingüísticas están sujetas a modificaciones entre culturas a pesar de la universalidad del fenómeno. En el caso de los estudiantes griegos de ELE, los malentendidos comunicativos se originan en una interpretación literal de las normas de cortesía, las cuales difieren de las de su lengua materna. A la luz de esta problemática, se enseñan fórmulas de saludos y despedidas a aprendices griegos de español entre los niveles A1-B1 mediante un corpus reducido de las fórmulas utilizadas en los telediarios. Asimismo, el análisis de su abordaje en 14 manuales entre los niveles A1-B1 revela una marcada simplificación y una contextualización insuficiente para su presentación. Por ello, la propuesta didáctica incentiva la reflexión sobre las estrategias comunicativas utilizadas en la lengua materna. Así, los estudiantes podrán adaptarse a los patrones discursivos del español (por ejemplo, la formalidad y la informalidad) y mejorar su competencia comunicativa.

Para finalizar, nos referimos a la investigación sobre las peticiones y la aceptación o rechazo de las invitaciones de Alonso Martín (2017). La autora trabaja con un grupo de estudiantes francófonos del ciclo terminale, es decir, los últimos dos años de la etapa lycée en el sistema educativo francés ${ }^{3}$. Así, conforma un corpus a partir de un cuestionario sobre sus creencias acerca del uso y aprendizaje de la cortesía en el aula de ELE, e incluye, además, un análisis de una muestra de manuales del ciclo mencionado. Asimismo, propone una serie de secuencias didácticas para practicar los actos de habla mencionados.

\footnotetext{
${ }^{3}$ Caballero Vega (2014, pp. 8-18) se focaliza en la enseñanza y la situación del español como lengua extranjera en Francia.
} 


\section{Sugerencias didácticas}

En este apartado, hacemos referencia a tres investigaciones que ofrecen descripciones teóricas de los actos de habla. Asimismo, si bien no constituyen secuencias o unidades didácticas completas y sistematizadas, incluyen una serie de interesantes recomendaciones para su enseñanza. En primer lugar, De Pablos-Ortega (2005) subraya la importancia de los aspectos sociopragmáticos en pos de evitar la creación de estereotipos erróneos de los hablantes de la lengua meta. Para la enseñanza del acto agradecer, sugiere los juegos de rol, la reflexión metapragmática contrastiva y el cine.

Por su parte, Garrido Rodríguez (2005), en su trabajo sobre la enseñanza de la cortesía y los actos de habla en el campo de ELE, enfatiza la relevancia de advertir a los estudiantes sobre los factores contextuales, al señalar si un determinado acto de habla puede considerarse cortés o no. La autora propone, asimismo, concentrarse en la conversación coloquial, explicar sus propiedades discursivas y delimitar los actos susceptibles de aparecer en este tipo de conversaciones. Finalmente, destaca la enseñanza del vínculo entre las respuestas prioritarias y las que no lo son, los actos de cortesía positiva y negativa, y las estrategias de mitigación e intensificación.

Por último, destacamos los aportes de Solís Casco (2005), quien en su trabajo sobre la enseñanza de los actos de habla indirectos, señala el valor de considerar la variación entre la lengua materna y la lengua meta del aprendiente, en cuanto a los elementos y estrategias utilizadas en los diferentes actos. La autora resalta la enseñanza de los aspectos pragmáticos que subyacen a la adecuación de los actos indirectos y la profundización en el estudio de los elementos que se encuentran en los diversos tipos de actos. Para ello, recomienda utilizar la publicidad y series de televisión y textos literarios que reflejen el habla coloquial.

La importancia de estos trabajos radica en que proporcionan lineamientos sumamente positivos desde un punto de vista didáctico ya que resultan factibles de ser incorporados al momento de diseñar actividades, propuestas o unidades enfocadas en distintos actos habla. Además, destacan la importancia de considerar los elementos contextuales, integrar los aspectos sociopragmáticos e incentivar la reflexión metapragmática. Asimismo, enfatizan la necesidad de utilizar actividades que se enfoquen en el uso de la lengua y que utilicen diversos recursos, como los medios, la publicidad y la literatura. De esta forma, se expondrá a los aprendientes a contextos donde podrán familiarizarse con los diversos actos de habla en situaciones atravesadas por factores de índole sociolingüístico y pragmático, a saber, consejos a colegas, compañeros de estudio, amigos, personas de diferentes grupos etarios y condiciones socioculturales.

\section{Conclusión}

A lo largo de esta investigación, hemos intentado ofrecer un panorama del recorrido de una serie de estudios que evidencian la situación de la enseñanza de la cortesía en el campo de ELE, atendiendo a sus corrientes teóricas y metodológicas y al análisis específico de los actos de habla. Nuestra revisión bibliográfica pone de 
manifiesto la riqueza que presenta, sobre todo en las últimas dos décadas, el campo de investigación sobre la enseñanza de la cortesía en general y los actos de habla en particular. Pudimos distinguir entre trabajos que se ocupan del abordaje de los actos de habla en manuales, propuestas didácticas focalizadas en su enseñanza, estudios que combinan los dos aspectos anteriores y, en menor medida, descripciones teóricas sobre los actos que incluyen sugerencias didácticas. La diversidad de investigaciones demuestra la creciente preocupación de los estudiosos de la didáctica de la cortesía por analizar su abordaje en los manuales. Los estudios sobre el tratamiento de los actos de habla en manuales evidencian, en general, que aún continúa siendo necesario investigar los modos de perfeccionar la incorporación de sus formas y funciones en los manuales y materiales de ELE. Asimismo, las unidades didácticas manifiestan la necesidad de diseñar materiales que atiendan a contextos y niveles más específicos.

Sobre la base de estas conclusiones y nuestras observaciones, es posible ofrecer una serie de sugerencias que, en nuestra opinión, pueden aportar a la didáctica de la cortesía y, particularmente, de los actos de habla. En primer lugar, consideramos que la enseñanza de los actos debe comenzar en los niveles iniciales y aumentar gradualmente en complejidad en los niveles posteriores. Por ejemplo, ha de empezar con actividades de reconocimiento hasta lograr la producción de enunciados corteses apropiados de acuerdo al contexto. Del mismo modo, no deben enseñarse solamente de forma explícita los aspectos pragmalingüísticos vinculados a los actos, sino también los elementos sociopragmáticos. En este sentido, los estudios basados en modelos sociopragmáticos y posiciones relativistas de la cortesía pueden realizar significativos aportes a su enseñanza. Además, los actos tienen que tratarse de manera uniforme y no priorizar la inclusión de unos actos por sobre otros.

Por otro lado, para remediar la carencia de información pragmática señalada en las diferentes investigaciones, las actividades deben incluir explicaciones más detalladas sobre los contextos de uso de los diferentes actos de habla y, además, promover la reflexión metapragmática. A su vez, no tienen que priorizar lo formal por sobre lo comunicativo y han de presentarse de forma gradual, contextualizada y sistematizada. De igual modo, es recomendable trabajar con actividades orales de a pares o en pequeños grupos, ya que brindan la oportunidad de interactuar y, de este modo, utilizar una mayor variedad de actos de habla. En estos casos, se ofrece la posibilidad de negociar y, por ende, se pueden activar no solo los elementos pragmalingüísticos, sino también los sociopragmáticos. Por último, como hemos señalado, resulta importante explotar recursos significativos como la televisión, las series, el cine y los recursos en internet, es decir, material que exponga a los estudiantes a input lingüístico asociado a diferentes elementos sociolingüísticos y pragmáticos.

Confiamos en que esta revisión sobre la enseñanza de la cortesía en el ámbito de ELE resulte una invitación estimulante para los estudiosos del área a enfocarse, por ejemplo, en el análisis de la incorporación de los actos de habla en manuales de los niveles más avanzados, ya que, como se evidencia en este trabajo, las investigaciones se centran generalmente en los manuales de los niveles iniciales. Asimismo, resultaría interesante continuar con la elaboración de propuestas y secuencias didácticas sobre otros actos de habla que se ajusten a diferentes niveles de referencia del MCER, a 
distintos espacios geográficos y a variedades del español diferentes a la peninsular. Del mismo modo, sería necesario trazar un panorama sobre el abordaje de otros aspectos de cortesía en la enseñanza de ELE, a saber, la atenuación, la intensificación y los marcadores discursivos. Sin duda, el desarrollo de estas líneas de investigación posibilitará y favorecerá su enseñanza.

\section{Referencias bibliográficas}

ALBELDA MARCO, M. (2005). El refuerzo de la imagen social en conversaciones coloquiales en español peninsular. La intensificación como categoría pragmática. En: BRAVO, D. (ed.) Estudios de la (des)cortesía en español. Categorías conceptuales y aplicaciones a corpora orales y escritos (pp. 93-118). Estocolmo, Buenos Aires, EDICE, Dunken.

ALONSO MARTÍN, C. (2017). El tratamiento de la cortesía con alumnado francófono en el aula de ELE: El caso de las peticiones y la aceptación o el rechazo de invitaciones [Tesis de maestría]. Santander, Universidad de Cantabria.

ARROYO MARTÍNEZ, L. (2014). Estudio de los actos de habla en el manual Destino Erasmus 1 y 2: La importancia de la competencia pragmática. En: N. CONTRERAS IZQUIERDO (ed.) Actas del XXIV Congreso Internacional ASELE. La enseñanza del Español como LE/L2 en el siglo XXI (pp. 99-106). Jaén, Asociación para la Enseñanza del Español como Lengua Extranjera.

BANI, S., y NEVADO, A. (2004). Aproximación a la cortesía verbal en manuales de E/LE. Artifara. Revista de lenguas y literaturas ibéricas y latinoamericanas, 4.

BARTOL MARTÍN, E. (2017). La enseñanza de la cortesía verbal en ELE: Análisis y actualización de contenidos [Tesis doctoral]. Salamanca, Universidad de Salamanca.

BLÁZQUEZ CASTILLO, A. (2015). La pragmática en el aula de ELE: la enseñanza de la cortesía verbal dirigida a estudiantes nativos de inglés. MarcoELE: Revista de Didáctica Español Lengua Extranjera, 21.

BRAVO, D. (2003). Actividades de cortesía, imagen social y contextos socioculturales: Una introducción. En: BRAVO, D. (ed.), Actas del Primer coloquio del programa EDICE «La perspectiva no etnocentrista de la cortesía: identidad sociocultural de las comunidades hispanohablantes» (pp. 98-108), Estocolmo, Universidad de Estocolmo.

BRAVO, D. (2009). Pragmática, sociopragmática y pragmática sociocultural del discurso de la cortesía: Una introducción. En: BRAVO, D., HERNÁNDEZ FLORES, N. y A. CORDISCO (ed.), Aportes pragmáticos, sociopragmáticos y socioculturales a los estudios de la cortesía en español, Buenos Aires, Dunken.

BRIZ GÓMEZ, A. (2005). El español coloquial, situación y uso. Madrid, Arco Libros.

BRODERSEN, L. M. (2019). El acto de habla «saludar» en los manuales de ELE/ELSE del español de la Argentina, Quintú Quimün, 3.

BROWN, P., y LEVINSON, S. C. (1987). Politeness: Some Universals in Language Usage. Cambridge, Cambridge University Press. 
CABALLERO DÍAZ, C. (2005). Pragmática e interculturalidad en la enseñanza del español como lengua extranjera. Las estrategias de cortesía en los manuales de E/LE [Tesis de maestría]. Alicante, Universidad de Alicante.

CABALLERO VEGA, B. (2014). La enseñanza de español como lengua extranjera en los últimos cursos de instituto en Francia. Análisis del manual Juntos y propuestas de unidades didácticas en relación con las cuatro nociones [Tesis de maestría], Oviedo, Universidad de Oviedo.

CALDERO CORNEJO, J. A. (2009). La cortesía en los actos de habla de especial importancia en el aprendizaje de E/LE [Tesis de maestría], Madrid, Universidad de Alcalá.

CONSEJO DE EUROPA. (2002). Marco común europeo de referencia para el aprendizaje, la enseñanza y la evaluación de lenguas. Aprendizaje, enseñanza, evaluación, Madrid, Ministerio de Educación, Cultura, Deporte, Instituto Cervantes y Editorial Anaya.

CORPAS PASTOR, G. (1997). Manual de fraseología española. Madrid, Biblioteca Románica Hispánica, Gredos.

DE PABLOS-ORTEGA, C. (2011). The pragmatics of thanking reflected in textbooks for teaching Spanish as a foreign language. Journal of pragmatics: 2411-2433.

DE PABLOS-ORTEGA, C. (2005). La enseñanza de aspectos sociopragmáticos en el aula de ELE. En: Álvarez, A. et al. (eds.) Actas del XVI Congreso de ASELE (Asociación de Español como Lengua Extranjera), Oviedo, Universidad de Oviedo: 515-519.

DERVIN, F., y LIDDICOAT, A. J. (2013). Linguistics for Intercultural Education. Ámsterdam, John Benjamins Publishing.

DUMITRESCU, D. (2006). Agradecer en una interlengua: Una comparación entre la competencia pragmática de los estudiantes nativos y no nativos del español en California, Estados Unidos. En: MURILLO, J. (ed.) Actas del Segundo Coloquio Internacional del Programa EDICE (pp. 375-406), Estocolmo, Universidad de Estocolmo.

ESCANDELL VIDAL, M.V. (2004). Aportaciones de la pragmática. En: SÁNCHEZ LOBATO, J. e I. SANTOS GARGALLO (Dirs.). Vademécum para la formación de profesores. Enseñar español como segunda lengua (L2) / lengua extranjera (LE) (pp. 179197). Madrid, SGEL.

FERNÁNDEZ LÓPEZ, M. DEL C. (2004). Principios y criterios para el análisis de materiales didácticos. En: Sánchez Lobato, J. e I. Santos Gargallo (Dirs.). Vademécum para la formación de profesores. Enseñar español como segunda lengua (L2) / lengua extranjera (LE) (pp. 715-734). Madrid, SGEL.

FERNÁNDEZ MARTÍN, P., y NÚÑEZ CORTÉS, J. A. (2017). Análisis comparado de actos de habla en manuales de ELE en los niveles A1 y A2. MarcoELE. Revista de Didáctica Español Lengua Extranjera, 24.

FERRER, M. C., y SÁNCHEZ LANZA, C. (2002). Interacción verbal: Los actos de habla. Rosario, Universidad Nacional de Rosario.

FRANCO MARTÍNEZ, A. (2014). Análisis de la competencia pragmática en estudiantes ELE: el arte de rechazar una petición [Tesis de maestría], Barcelona, Universitat de Barcelona; Universitat Pompeu Fabra Barcelona. 
GARRIDO RODRÍGUEZ, M. DEL C. (2005) Cortesía y actos de habla en la enseñanza de E/LE. La competencia pragmática y la enseñanza del español como lengua extranjera. En: ÁlvarEZ, A. et al. (eds.) Actas del XVI Congreso de ASELE (Asociación de Español como Lengua Extranjera) (pp. 308-317). Oviedo, Universidad de Oviedo.

GRICE, H. P. (1975). Logic and conversation. En: COLE, P. y J. MORGAN (eds.). Syntax and Semantics II: Speech Acts (pp. 78-113). Nueva York, Academic Press.

GRIMM, A. (2012). El tratamiento de la cortesía en el PCIC aplicado a la enseñanza de ELE a germanohablantes. [Available at http://www.academia.edu/10652451/]

HAVERKATE, H. (1994). La cortesía verbal. Estudio pragmalinguístico. Madrid, Gredos.

JIMÉNEZ, D., MAZA, M., MILEWSKA, J., y MORA, J. P. (2016). Aplicación de la pragmática en clase. Propuesta para enseñar las disculpas en lengua materna, segunda lengua y lengua extranjera. Doblele: revista de lengua y literatura, $N .^{\circ} 2: 24-35$.

KASPER, G., y ROSE, K. R. (2002). Pragmatic Development in a Second Language, Language Learning: A Journal of Research in Language Studies, 52, 1.

LEECH, G. N. (1983). Principles of Pragmatics. Essex, Longman.

LÓPEZ VILA, H. (2016). La cortesía verbal valorizante en clase de ELE [Tesis de maestría], Logroño, Universidad Internacional de La Rioja.

MARTÍN AIZPURU, L. (2010). Las fórmulas rutinarias sociales en la clase de Español como Lengua Extranjera (ELE), Lenguas Modernas, 36, 65-84.

MARTÍN AIZPURU, L., y PINEDA MORALES, L. (2011). Los piropos en el aula de E/LE, Revista de Lingüística y Lenguas Aplicadas, 6, 261-273.

MARTÍNEZ GUILLÉN, D. (2018). Análisis de la cortesía a través de la observación de películas [Tesis de maestría]. Universidad Nacional de Educación a Distancia.

MIRANDA UBILLA, H. (2000). La cortesía verbal en textos para la enseñanza del español e inglés como lenguas extranjeras [Tesis doctoral], Madrid, Universidad de Valladolid.

MURILLO MEDRANO, J. (2004). La pragmática y la enseñanza del español como segunda lengua, Educación, 28 (2), 255-267.

PALEARI, V. (2015). De la investigación a la clase de E/LE: una propuesta sobre la cortesía verbal en la divergencia de opinión entre italianos y españoles. MarcoELE: Revista de Didáctica Español Lengua Extranjera, 21.

PATTERSON, J. (2015). La pragmática aplicada a la competencia comunicativa en español. Estudio sobre la cortesía en cuatro manuales de español como lengua extranjera [Tesis de maestría], Gotemburgo, Universidad de Gotemburgo.

PIATTI, G. (2003). La cortesía: Un contenido funcional para los programas de español como lengua extranjera. En: BRAVO, D. (ed.). Actas del Primer coloquio del programa EDICE «La perspectiva no etnocentrista de la cortesía: identidad sociocultural de las comunidades hispanohablantes» (pp. 355-397). Estocolmo, Universidad de Estocolmo.

RAMAJO CUESTA, A. (2013). La secuencia del cumplido en los manuales de ELE y de árabe dialectal como lengua extranjera. Revista Nebrija de Lingüística Aplicada a la Enseñanza de Lenguas, 13. 
RAMOS GONZÁLEZ, N. (2015). La expresión de la cortesía en el aula de ELE: una propuesta didáctica para evitar el fallo pragmático a partir del análisis de las series de televisión españolas en internet [Tesis doctoral], Granada, Universidad de Granada.

RAMOS GONZÁLEZ, N. M., y RICO MARTÍN, A. M. (2014). Análisis de la expresión de la cortesía en RTVE Internacional para la enseñanza de Español Lengua Extranjera. RLA. Revista de lingüística teórica y aplicada, 52(1), 79-103.

RODRÍGUEZ SANTOS, J. M. (2017). El acto de habla como unidad de comunicación en el aprendizaje de una segunda lengua: Tratamiento en materiales didácticos de E/LE. Tonos Digital, 32.

RODRÍGUEZ SANTOS, J. M., y MIRÓ MARTÍ, O. (2018). Imagen y (des) cortesía: Interferencias sociopragmáticas y efectos en la comunicación en algunos manuales de ELE de Corea del Sur. Revista Internacional de Lenguas Extranjeras/International Journal of Foreign Languages, 8, 91-109.

ROMERO BETANCOURT, M. V. (2012). Adquisición de pragmática en segunda lengua: Un modelo didáctico para la enseñanza de la pragmática [Tesis de maestría] Indiana University.

SEARLE, J. R. (1969). Speech Acts: An Essay in the Philosophy of Language. Cambridge, Cambridge University Press.

SEARLE, J. R. (1975). Indirect speech acts. En: COLE, P. y J. MORGAN (eds.): From syntax to semantics, Nueva York, Academic Press, 59-82.

SEOANE, E., y MURIAS, R. (2012). Así lo digo y así lo hago: Una propuesta didáctica para la enseñanza de gestos y fórmulas rutinarias sociales en la función comunicativa del saludo. En: MENÉNDEZ SÁNCHEZ, F. (ed. y coord.). Boletín ELE Anatolia, Dirección de Programas Educativos en Turquía. Embajada de España, 78-91.

SOLÍS CASCO, I. M. (2005). La pragmática en el aula de ELE: Los actos de habla indirectos. La competencia pragmática y la enseñanza del español como lengua extranjera. En: ÁLVAREZ, A. et al. (eds.) Actas del XVI Congreso de ASELE (Asociación de Español como Lengua Extranjera) (pp. 607-615). Oviedo, Universidad de Oviedo.

TRIGO CAMPELO, R. (2017). ¿Cortés yo? ¡Y un jamón! Análisis de la evolución del tratamiento de la cortesía en manuales de ELE de nivel superior [Tesis de maestría], Barcelona, Universitat de Barcelona; Universitat Pompeu Fabra Barcelona.

URBINA VARGAS, M. S. (2009). Una propuesta pedagógica para el desarrollo de la competencia pragmática en español como segunda lengua o lengua extranjera. Innovaciones educativas, 11(16), 8.

VALLS ANGUERA, C. (2012). «Ay, perdona, no quería molestarte». Enseñar y aprender a disculparse en español. MarcoELE. Revista de Didáctica Español Lengua Extranjera, 15, 1-64.

ZERVA, A. (2013). Los principios de cortesía en la enseñanza del español a aprendices griegos: Las fórmulas de saludos y despedidas. Foro de Profesores de E/LE, 9, 153-160. 